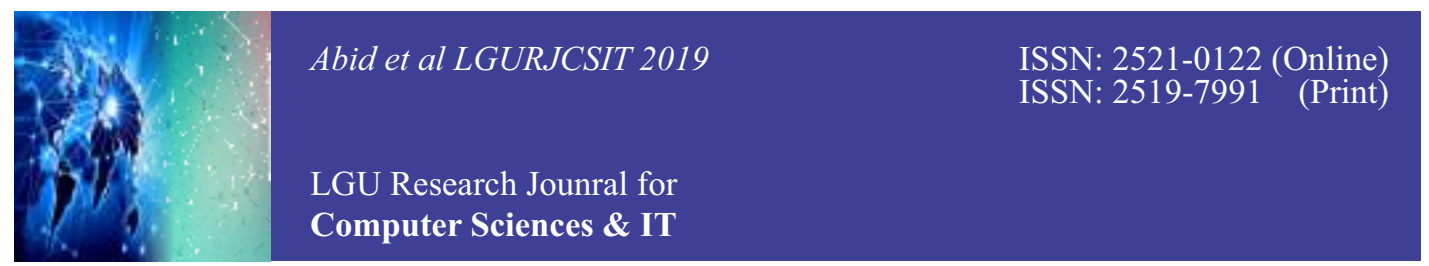

Vol. 3 Issue 2, April - June 2019

\title{
Flying Ad Hoc network (FANET) Communication between UAV's
}

Saman Abdi ${ }^{1}$, Muhammad Javed ${ }^{2}$

${ }^{1}$ Lahore Garrison University, ${ }^{2}$ University of south Asia

\begin{abstract}
:
In FANET network communication is one of the major concern, and also communication between multi-UAV frameworks. The communication is established between UAV's and base terminal through satellite or any other structure. This kind of communication create issue between UAV's, the long distance between UAV's can create blackouts in communication, because of these issues the ad hoc network can be used among the UAV's, this kind of communication network is called FANET. In this paper I will discuss the implementation of the network and how the network structure can provide reliable communication ad transfer of data between the unnamed aerial vehicles UAV's and the base terminals.
\end{abstract}

Keywords: Unmanned Ariel Vehicles (UAV), flying Ad Hoc network (FANET), Multi UAV systems (MUAV)

\section{INTRODUCTION}

Improvements in sensors and in electric components, the manufacturing of Unmanned Ariel vehicles (UAVs) has turned out to be conceivable. In any case, the task capacity and adaptability of one substantial UAV is constrained. Along the progression of inserted frameworks and decrease of miniaturized scale electromechanical frameworks, rather than a solitary extensive UAV. The collaboration of numerous little UAVs is guaranteed. Therefore, the making of the frameworks past the capacity of a UAV has turned out to be conceivable and this has ended up being to be extremely profitable. Correspondence is a standout amongst the most imperative issues which was experienced when attempting to guarantee the interaction of multi UAVs frameworks. One of the techniques created to take care of the issue of correspondence Unmanned Ariel Vehicle is the ad-hoc network [1]. A specially appointed communication unit is a circulated remote communication structure that permits correspondence hubs without the requirement for framework [2]. In this investigation, contemplates on the usage of ad-hoc network engineering test condition are exhibited.

If the quantity of UAVs utilized in similar frameworks increment, the plan of a proficient network engineering turns into a vital issue. The network engineering structures, appeared at Figure 1, are created to take care of the correspondence issue among numerous UAVs [3]. As per Figure 1(a), all UAVs must be joined to just a single base terminal. In any case, when this engineering is utilized, the task territory is constrained to the correspondence go among UAVs and the base terminal. With the end goal to understand the range limitation issue, UAVs can be associated with a satellite rather than a base terminal. In any case, for this situation, the expense would be high and the overwhelming satellite correspondence equipment would should be installed to each and every UAVs. Then again, this framework won't have the capacity to be utilized in some specific circumstances, for example, outrageous climate conditions. Another correspondence design is to utilize in excess of one base terminal for multi UAV frameworks, as in Fig 1(c). Anyway numerous base terminal design won't be reasonable for military and fiasco application situations. Since the foundation can't be 
confided in such a circumstance. Another correspondence design is FANET, which is appeared in Fig 1(d). The work of this engineering sets up specially appointed network among UAVs and along these lines can take care of the correspondence extend confinement issue. As indicated by this design, while some UAVs speak with the base terminal or by means of satellite, different UAVs with no immediate correspondence with base terminal can utilize their correspondence by means of ad hoc network structure. The most vital favorable position of flying impromptu networks is their capacity to give ongoing correspondence without need of any framework.
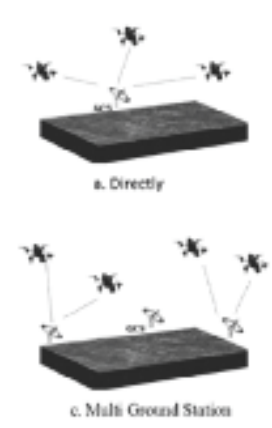

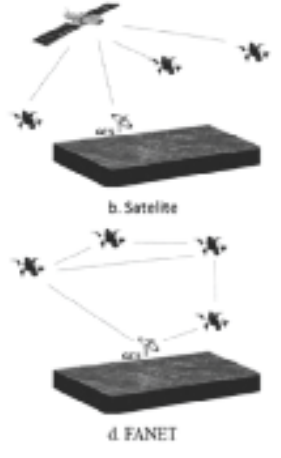

A FANET
Fig. 1 communication structure of UAV's.

FANETs are considered as an alternate specially appointed ss hoc network infrastructure as restricted MANETs or VANETs because of their exceptional highlights [1]. Imperative contrasts between current specially appointed networks and FANETs are recorded as pursues:

Mobile man is the hub of a MANET while moving vehicle is the hub of a VANET. Same like that UAV's are the hub of FAET network. Be that as it may, the ad hoc network hubs are UAVs and UAVs can achieve a lot higher paces. Therefore, FANET hubs move quicker than MANET or VANET hubs. Contingent upon the high versatility.

All the interaction protocols should be updated. Hubs at FANETs which guarantee correspondence among UAVs is additionally vital. Notwithstanding, the most essential motivation to utilize multi-UAV frameworks is to gather data from nature. Along these lines just like the case for remote sensor networks (WSN) and in addition, they can both transfer the gathered information to a server farm.

MANET hubs and VANET hubs should be designed to be at appropriate distance to function properly otherwise blackouts can occur in communication among UAVs.

Frameworks for more than one UAV, loads of various sensors are clear. Every sensor can have an alternate correspondence necessity. In this circumstance FANET ought to have the adaptability to give diverse prerequisites.

The principle inspiration of this examination is to guarantee the acknowledgment of a FANET structure, which have picked up notoriety around the globe lately [4-6]. This proving ground is acknowledged with minimal effort and effectively accessible equipment.

\section{FANET}

Best communications way to base terminal and transferring data to base terminal for multi UAV is FANET network.

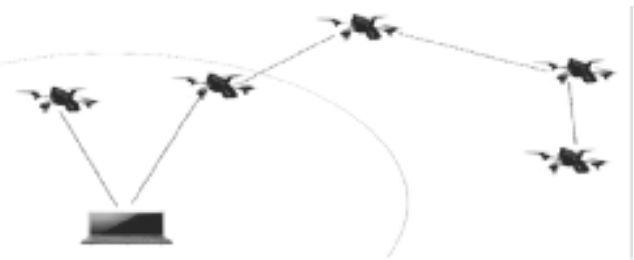

Fig. 2. Extended communication between multi-UAV systems.

Fig. 2 shows particular UAVs are closet to the base terminal, different nodes can be communicated through FANET. Because the ones connected with base terminal can transmit data to and from the ones which are out of the rage of base terminal. Models of FANET use situations which are introduced underneath.

\section{A. Relaxed Communication without restrictions}

All UAVs in the framework can be associated with a solitary base terminal. Anyway for this situation the activity area is restricted to the scope of UAVs with base terminal. Likewise with ad-hoc network, the scope framed infrastructure isn't restricted to the scope of base terminal because of the way that UAVs that can't set up an immediate connection with the base terminal however can speak with the UAV base terminal by means of different UAVs.

\section{B. Communication Reliability in $U A V S$}

UAV frameworks can work in profoundly powerful conditions. For instance, an undertaking may change amid task and 
furthermore, an association between the base terminal and a UAV might be intruded on amid activity because of the antagonistic climate changes. As appeared in Fig.3, association between detached UAV from the beginning, different UAVs is kept up multi-UAV frameworks engineering bolsters ad hoc network. Unwavering quality of multi-UAV frameworks is expanded with availability [1].

\section{Communication Association}

Little and smaller than normal UAVs are extremely light weighted. In spite of the restricted capacities of single UAV, more perplexing undertakings by multi-UAV frameworks. Synergistic activities of UAVs require ceaseless interaction with one another. Be that as it may, because of the restricted load limit of the UAVs, they will be unable to convey overwhelming Interaction equipment to empower Interaction between the UAV and the base terminal. FANET design can be utilized to build up an association between the smaller than normal UAVs with lighter and less expensive equipment. Impact can be stayed away from and furthermore FANET engineering can give coordination between UAVs with the end goal to finish the mission effectively.

\section{Less weight and Less expensive with FANET}

Weight issue isn't legitimate for little UAVs. In the meantime, in high-elevation and low-perseverance UAVs, weight is critical. Fewer weight implies higher height and longer perseverance [7]. In the event that the Interaction design of the multi-UAV frameworks will be founded on totally base terminal and UAV, UAVs must convey a generally heavier Interaction equipment for communicating.

On the off chance that FANET is utilized in multi-UAV frameworks, associations are set up among a particular UAV and the UAV base terminal and different UAVs are additionally ready to speak with one another through FANET. At the point when FANET is utilized along these lines, the sturdiness of multi-UAV frameworks can be expanded.

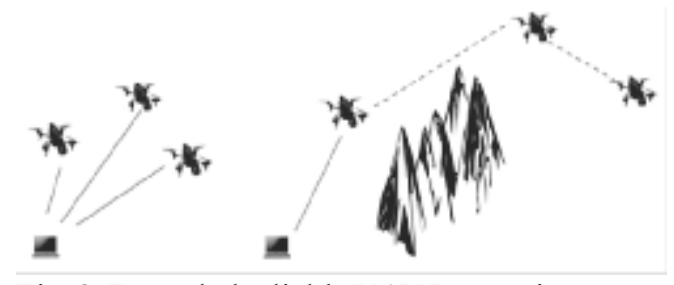

Fig. 3. Extended reliable UAV Interaction.

\section{FANET IMPLEMENTATION}

The primary goal to this investigation is to understand the execution of test bed with minimal effort for FANETs. Because of its ease, simple organization and simplicity of auxiliary improvement, AR.D 2 is utilized as the UAV stage [8]. 802.11 can control Ar D.

Wireless network module can setup association between Ar.D. It can't be utilized for building up a specially appointed network between UAVs. With the end goal to work a specially appointed network between Ar D. Then again, monitoring Ar.D is another issue that must be dealt with. On the off chance that Ar.D is controlled with an immediate Wi-Fi association with a focal terminal (like a work terminal or tablet), the subsequent structure can't be utilized. UAVs can be controlled regardless of whether ground base and the UAVs are out of the communication. Luckily, maker of Ar D, provides open APIs to create user design programming. Open source code was used to keep running on Raspberry Pi to operate Ar Ds. We incline toward NodeJs because of source Ar D control programming [11]. NodeJs started to create in 2009 an enrolled symbol of Joyent organization.

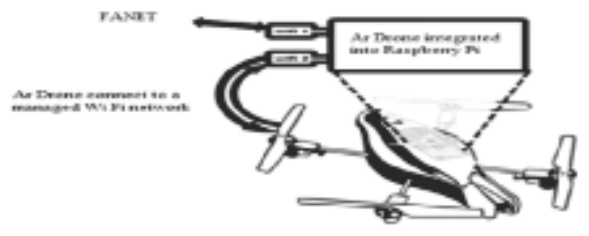

Fig. 4. Raspberry Pi \& Ar D implementation.

NodeJs stage that is based on superior JavaScript motor. For the checkpoint situation executed in test condition, we utilized code parts that composed Andrew Nesbitt in NodeJs dialect [12]. Be that as it may, it was found to work unsteadily through tests investigations. Important remedies executed in code.

Wi-Fi association can be used for controlling Ar D. Control guidelines are produced with controlling gadget (presumably a PC or tablet) and the directions are sent to Ar D with a UDP association. In our FANET test bed, control directions are created by Raspberry Pi. In any case, they should be sent with a Wi-Fi association. As previously stated, we utilized one Wi-Fi dongle for specially appointed network foundation among UAVs, and Ar D Wi- 
Fi does not bolster impromptu mode. It reasons that we require extra $\mathrm{Wi}-\mathrm{Fi}$ association in Raspberry $\mathrm{Pi}$. The other Wi-Fi association is used for directing Ar D with the goal that explore the set checkpoints.

Portrayal of ad hoc network is shown Fig 4 have completed are exhibited Fig 5. Ar D are attached with Raspberry Pi. Framework that provides flight recorder ad transfer data to UAV [13]. Wi-Fi is utilized to build up communication between other Raspberry Pi. Raspberry Pi send control guidelines over Wi-Fi association.

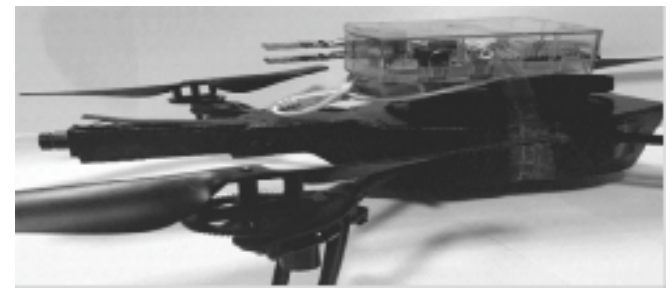

Fig. 5. Raspberry Pi and Ar D Structure.

\section{FUTURE WORK}

The future work includes, calculation of empirical data for this purposed network, Efficient modules for connecting UAV's with each other and with base station. Long range network architectural design to connect UAV's.

\section{CONCLUSION}

Small UAVs can accomplish way more than big UAVs by using the right components to fulfill their mission. A lot of Interaction issue have been pointed out and discussed which are vital for structure, between UAVs. FANET that is fit for fathoming communication go limitations among. Likewise, a ceaseless stream of data through these structures is additionally guaranteed. This investigation introduces a savvy and effectively repeatable test condition for execution; to help FANET inquire about examinations. Taking everything into account, it has been exhibited that the FANET framework is a minimal effort framework that can be utilized in all colleges to understand the test condition.

\section{References}

[1] Ilker Bekmezci, Ozgur Koray Sahingoz and Samil Temel, "Flying Ad-Hoc Networks (FANETs): A survey", Ad Hoc Networks 11(3):1254-1270, 2013
[2] C. Siva Ram Murthy and B.S. Manoj, "Ad Hoc Wireless Networks: Architectures and Protocols", Prentice Hall - ISBN-10: 0133007065,2012

[3] E.W. Frew and T.X. Brown, "Networking issues for small unmanned aircraft systems", Journal of Intelligent and Robotics Sysstems 54 (1-3), 2009.

[4] Ozgur Koray Sahingoz,'Networking Models in Flying Ad-Hoc Networks (FANETs): Concepts and Challenges", Journal of Intelligent \& Robotic Systems 74(1-2), 513-527,2014

[5] Stefano Rosati, Karol Kruzelecki, Gregoire Heitz, Dairo Floreano and Bixio Rimoldi, "Dynamic Routing for Flying Ad Hoc Networks", 2014

[6] Juergen Eckert, David Eckhoff and Reinhard German, "Flying Ad-Hoc Network Communication for Detecting Thermals: Feasibility and Insights",2013

[7] J. Clapper, J. Young, J. Cartwright and J. Grimes, "Unmanned Systems Roadmap", Tech. Rep. Dept. OfDefense, 2007.

[8] Ar D 2.0, http://ardrone2.parrot.com/, 06.02.2015.

[9] $\mathrm{R}$ a s s b e r r y $\quad \mathrm{P}$ i , http://www.raspberrypi.org, 09.02.2015.

[10] Byzantium Project ByzPi, http://byzantium.github.com/ByzPi/, 11.02.2015.

[11] NodeJs. http://www.nodejs.org/, 13.02.2015.

[12] Byzantium Project ByzPi, http://byzantium.github.com/ByzPi/, 16.02.2015.

[13] Flight Recorder for Ar.Drone 2.0, http://ardrone2.parrot.com/apps/flightrecorder/, 19.02.2015. 